\title{
A Case Report of Osteosarcoma of the Skull
}

\author{
Bouali Sofiene*, Bouhoula Asma, Boubaker Adnene, Ben Said Imed, Kallel Jalel, \\ Aouij Lassaad, Jemel Hafedh \\ Department of Neurosurgery, Faculty of Medicine, University of Tunis El Manar, National Institute of \\ Neurology Mongi Ben Hmida, Tunis, Tunisia \\ Email: ${ }^{*}$ sofienebouali@hotmail.fr
}

Received 6 April 2014; revised 6 May 2014; accepted 14 May 2014

Copyright (C) 2014 by authors and Scientific Research Publishing Inc.

This work is licensed under the Creative Commons Attribution International License (CC BY).

http://creativecommons.org/licenses/by/4.0/

c) (7) Open Access

\begin{abstract}
Background: Osteosarcoma is the most common primary malignancy and aggressive neoplasm of bone composed of spindle cells producing osteoid. The incidence of primary osteogenic sarcomas of the skull is about $1 \%$ to $2 \%$ of all skull tumors. Methods: We present an extraordinary case of osteosarcoma arising in the postero-temporal region of the skull. The clinical features, diagnosis and management are discussed. Results: A 34-year-old woman was operated in our department for mass, fixed to bone in the left postero-temporal area. Histopathology confirmed the diagnosis of osteogenic sarcoma. Conclusion: We review the literature of reported cases of primary osteogenic sarcomas of the skull to discuss the common clinical presentation, evaluation methods, and recommended treatment plans.
\end{abstract}

\section{Keywords}

Osteosarcoma, Primary Tumor, Skull

\section{Introduction}

Osteosarcoma (OS) of the skull is an extremely rare tumour particularly as a primary tumor. Osteosarcomas are typically long bone tumors and rarely affect the skull particularly after radiation or chemotherapy for other neoplasms. The incidence of primary osteogenic sarcomas of the skull is about $1 \%$ to $2 \%$ of all skull tumors [1] [2]. Reports are few and most combine skull lesions with the much more frequent Osteosarcoma of the maxillo-mandibular complex [3].

We report a rare case of Osteosarcoma arising in parieto-temporal bone.

\section{Case Report}

A 34-year-old woman presented with a 4-year history of a postero-temporal bump which had rapidly enlarged in "Corresponding author. 
5 months prior to admission. Physical examination demonstrated a $5 \times 7 \mathrm{~cm}$ rubbery hard, non tender mass, fixed to bone in the left postero-temporal area. The remainder of physical and neurological evaluations was normal, as were all routine laboratory studies.

Both brain computerized tomography (CT) CT-scan and Magnetic resonance imaging (MRI) showed an extra and intra axial lesion which had three components: fleshy, cystic and ossified (Figure 1 and Figure 2).

The patient underwent surgery with a complete resection of the lesion associated with dural plasty and cranial reconstruction (Figure 3 and Figure 4). Postoperative CT scan showed gross total removal (Figure 4).

Histopathology exam confirm the diagnosis of osteoginec sarcoma (Figure 5). Postoperative radiation therapy is ongoing.
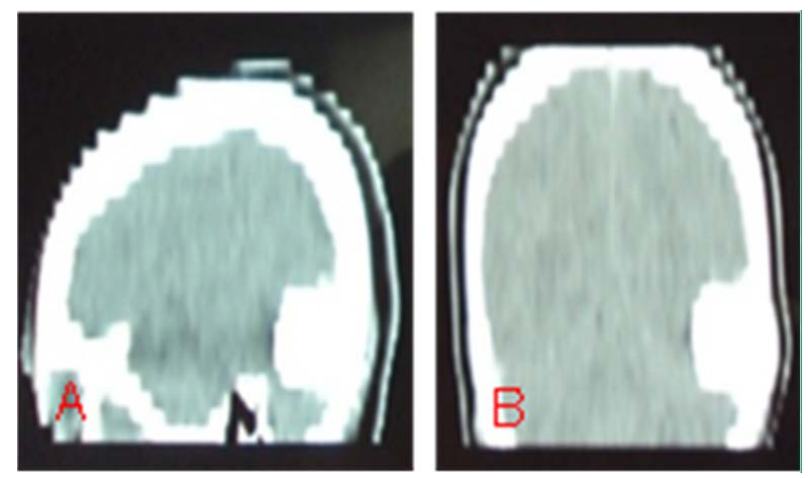

Figure 1. Axial CT scan showing a left postero-temporal lesion made 4 years ago.
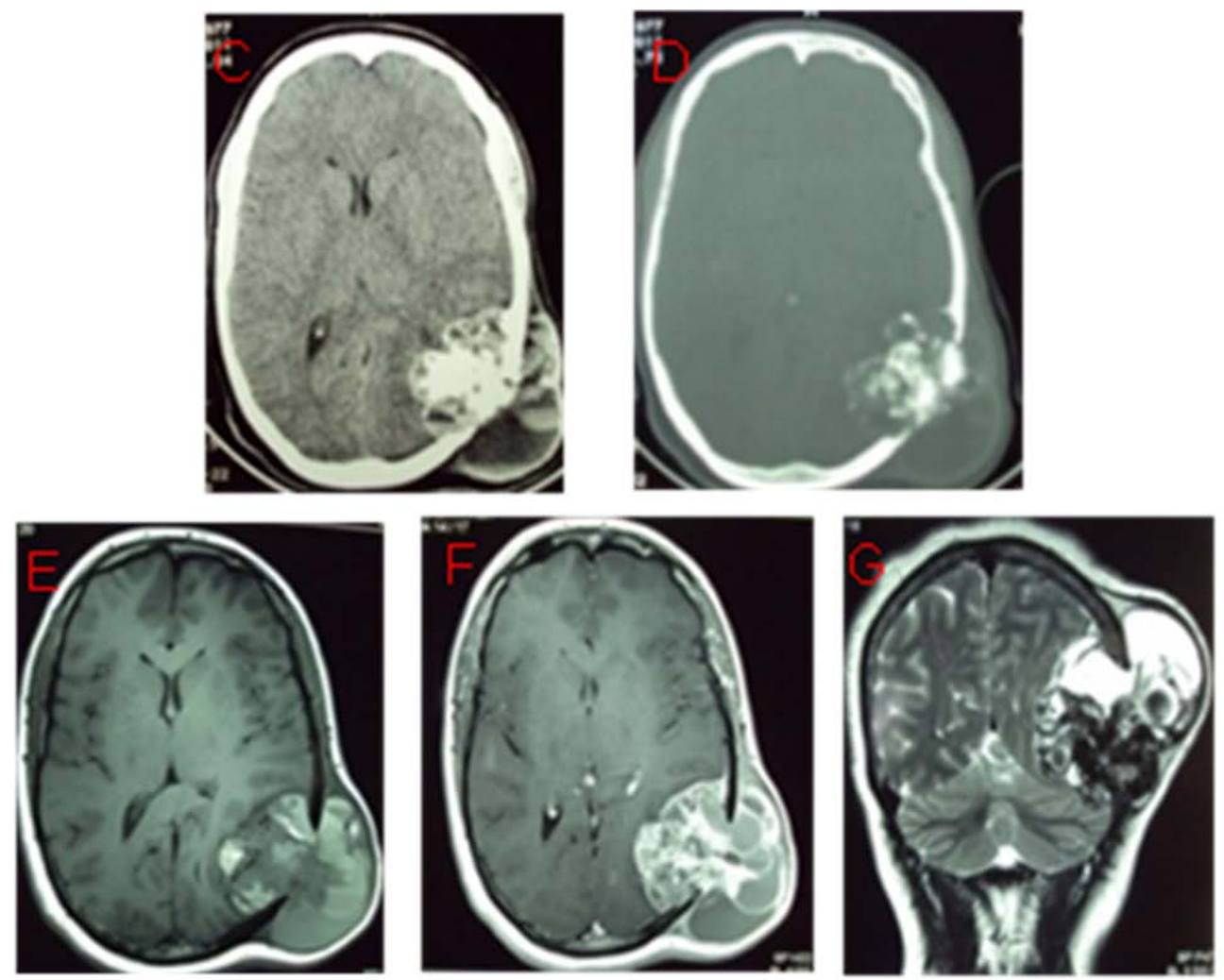

Figure 2. axial CT scan (C and D) without contrast, showed irregular calcification and low attenuation areas (bone window setting). MRI showed a large mass arising from the postero-temporal aspect of the skull on the left side. There was a calcified hemorrhagic part of the mass with marked vascularity of the tumor ( $\mathrm{E}, \mathrm{F}$ and $\mathrm{G})$. 

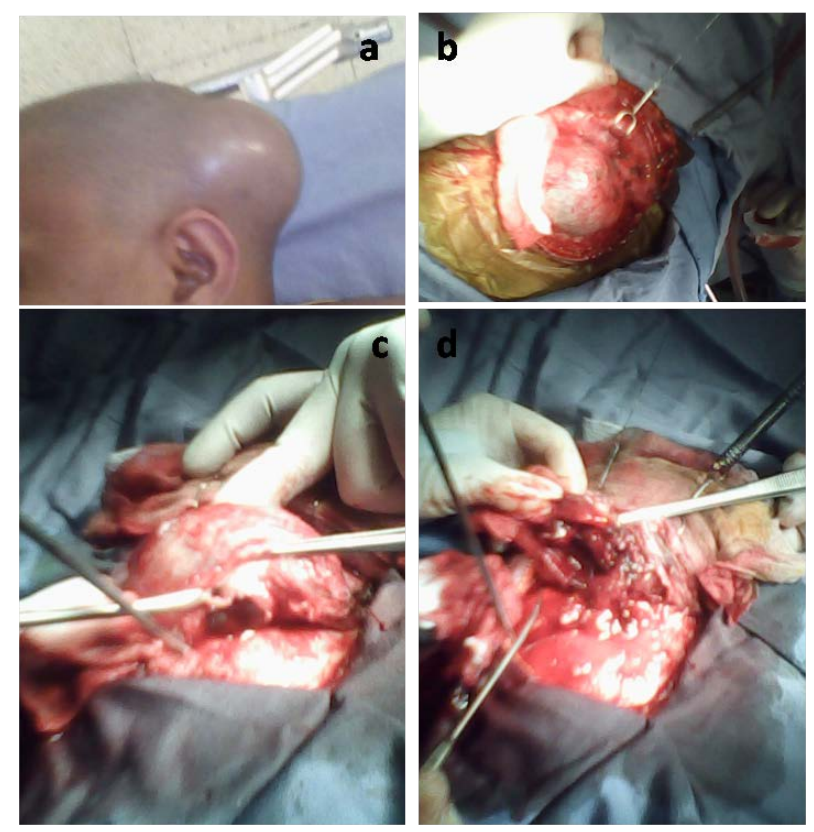

Figure 3. Pre-operative (a) and per-operative (b, c and d) pictures showing the cutaneous aspect and the different time of surgery.
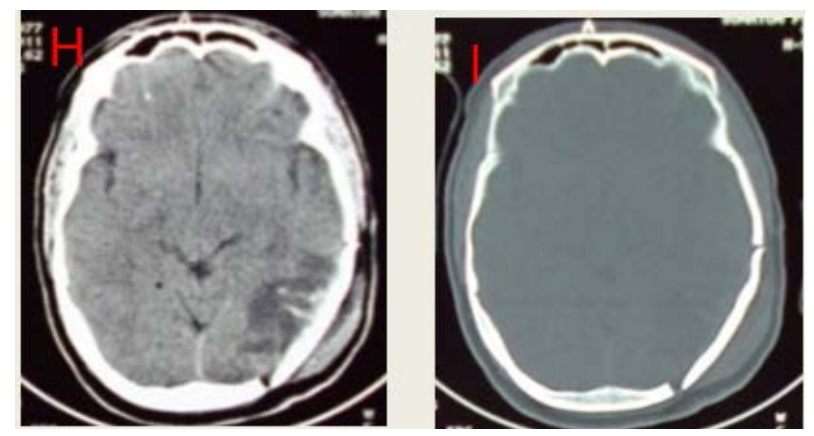

Figure 4. Post operative axial ct scan (H and I).

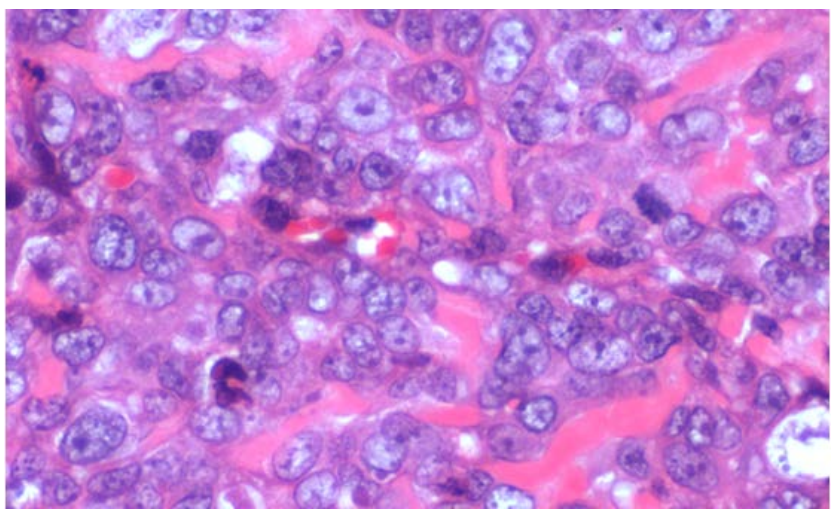

Figure 5. Sections were subjected to Hemotoxylin \& Eosin staining, showing the presence of pleomorphic spindeloid to polygonal cells with numerous atypical mitoses. Separating the neoplastic cells are seams of a bright pink matrix which is typical of osteoid and typical of an osteosarcoma. 


\section{Discussion}

Osteosarcoma is the most common primary malignancy of bone and 5\% to $6 \%$ arise in the craniofacial bones [1] [4]. The etiology and precise pathogenesis of this disease remain unknown [1]. Osteogenic sarcoma is largely a disease of the young but about one-third occurs in patients over 40 years of age [2] [5]. They are more common in male than female and the vast majority of craniofacial Osteosarcomas are located in the zygomatic bone [6] [7]. They are an aggressive neoplasm composed of spindle cells producing osteoid [8]. Clinical features are localized pain that frequently begins after injury.

Common presenting symptoms were swelling, pain, or both and the most important finding in physical examination is a soft tissue mass, which is frequently large and tender to palpation. Radiological investigations include CT-scan and MRI. The general radiographic features are not specific but may be osteolytic, osteoblastic or mixed [1]. The spicular pattern of calcification is usually present and no radiographic finding is pathognomonic [1] [2]. The final diagnosis of Osteosarcoma was only established by the histological studies [8].

It can be classified into three main subtypes: the chondroblastic, osteoblastic, and fibroblastic types [7] [8].

The recommended treatment has not yet been established, but radical surgery for extirpation including a large margin of normal bone is the most significant factor contributing to a good outcome. Adjuvant therapy, such as irradiation or chemotherapy can be performed if radical resection cannot be achieved. The role of chemotherapy is less clear for cranial tumors [6] [7].

The 10 years survival following resection of large tumors was $69 \%$ if the resection was complete but was only $13 \%$ if excision was incomplete [1] [2].

\section{Conclusion}

Because of the rarity of this type of tumor and a very poor prognosis, treatment of Osteosarcomas has not yet benefited from a rigorous evaluation and needs more studies to research the role of chemo and radiotherapy.

\section{Conflicts of Interest}

All authors declare no conflict of interest.

\section{References}

[1] Benson, J.E., Goske, M., Han, J.S., et al. (1984) Primary Osteogenic Sarcoma of the Calvaria. American Journal of Neuroradiology, 5, 810-813.

[2] Bose, B. (2002) Primary Osteogenic Sarcoma of the Skull. Surgical Neurology, 58, 234-240. http://dx.doi.org/10.1016/S0090-3019(02)00864-9

[3] Kanazawa, R., Yoshida, D., Takahashi, H., et al. (2003) Osteosarcoma Arising from the Skulle Case Report. Neurologia Medico-Chirurgica, 43, 88-91. http://dx.doi.org/10.2176/nmc.43.88

[4] Sturgis, E.M. and Potter, B.O. (2003) Sarcomas of the Head and Neck Region. Current Opinion in Oncology, 15, 239252. http://dx.doi.org/10.1097/00001622-200305000-00011

[5] Chennupati, S.K., Norris, R., Dunham, B. and Kazahaya, K. (2008) Osteosarcoma of the Skull Base: Case Report and Review of Literature. International Journal of Pediatric Otorhinolaryngology, 72, 115-119. http://dx.doi.org/10.1016/j.ijporl.2007.08.015

[6] Chang, C.S. (2010) Craniofacial Reconstruction of Primary Osteogenic Sarcoma of the Skull. Journal of Plastic, Reconstructive \& Aesthetic Surgery, 63, 1265-1268.

[7] Saeter, G. (2005) Minimum Clinical Recommendations for Diagnosis, Treatment and Follow-Up of Osteosarcoma. Annals of Oncology, 16, 71-72. http://dx.doi.org/10.1093/annonc/mdi822

[8] Fukunaga, M. (2005) Low-Grade Central Osteosarcoma of the Skull. Pathology-Research and Practice, 201, $131-135$. http://dx.doi.org/10.1016/j.prp.2004.12.001

[9] Maurizio, S. (1994) Radiation-Induced Osteosarcomas of the Skull: Report of Two Cases and Review of the Literature. Clinical Neurology and Neurosurgery, 96, 226-229. http://dx.doi.org/10.1016/0303-8467(94)90073-6 


\section{Abbreviations}

CT: Computed Tomography MRI: Magnetic Resonance Imaging

OS: Osteosarcoma 